\title{
Carotid Intima-Media Thickness and Carotid Plaques Improves Prediction of Obstructive Angiographic Coronary Artery Disease in Women
}

\author{
Ana Teresa Timóteo, MD', Miguel Mota Carmo, MD, PhD', and \\ Rui Cruz Ferreira, MD'
}

\begin{abstract}
Does carotid intima-media thickness (cIMT), a surrogate marker of cardiovascular events, have predictive incremental value over established risk factors for stable coronary artery disease (CAD)? Prospective study of 300 patients, with suspected stable CAD, admitted for an elective coronary angiography and carotid ultrasound. The CAD patients had a higher cIMT, which showed a modest predictive accuracy for CAD (area under the receiver-operating characteristic curve 0.638, 95\% confidence interval 0.576-0.70I, $P<.00$ I). The cIMT was an independent predictor of CAD, together with age, gender, and diabetes. C-statistic for CAD prediction by traditional risk factors was not significantly different from a model that included cIMT, carotid plaque presence, or both. However, in women, it was significantly increased by the addition of cIMT or carotid plaque presence. Although cIMT cannot be used as a sole indicator of CAD, it should be considered in the panel of investigations that is requested, particularly in women who are candidates for coronary angiography.
\end{abstract}

\section{Keywords}

carotid intima-media thickness, coronary artery disease, cardiovascular risk prediction

\section{Introduction}

Cardiovascular diseases are the leading cause of death in developed countries. ${ }^{1}$ They are also a major problem in Portugal, not only as far as coronary artery disease (CAD) is concerned but also in terms of stroke, the incidence of which is among the highest in the world. ${ }^{2}$ In fact, in developed countries, the most common cardiovascular disease is CAD, which is also the leading cause of death in all the European Union with the exception of Greece, the former Yugoslav Republic of Macedonia and Portugal, where it is stroke. ${ }^{2}$ It is thus important to identify patients at risk of developing cardiovascular events to enable preventive interventions and promote lifestyle modifications. Traditional risk prediction scores have proven very useful in identifying persons at risk of coronary heart disease, but such tools have limitations. Carotid intima-media thickness (cIMT) is a well-described surrogate marker for cardiovascular disease that it is easily obtained by a noninvasive test and increased cIMT has been associated with prevalent and incident CAD and stroke. ${ }^{3-6}$ Furthermore, plaque presence, which has been shown to be associated with coronary heart disease independent of cIMT measurement in several studies, seems to improve risk prediction. ${ }^{7,8}$ However, most studies addressed the relationship between cIMT and acute cardiovascular events in the followup. ${ }^{5,6}$ Fewer studies analyzed the relationship between cIMT and angiographic coronary artery disease - a stable end point with different pathopysiological mechanisms.

In the present study, we aimed to investigate the relationship between cIMT and angiographic CAD in patients evaluated by coronary angiography for suspected CAD. We intended to provide more information to help answer two important questions. Is cIMT evaluation useful in clinical practice? Does it help to define indications for coronary angiography?

\section{Population and Methods}

The present study is an observational and cross-sectional study, with prospective inclusion of patients admitted for an elective coronary angiography with suspected CAD (with stable angina and/or with documented ischemia on noninvasive tests). All patients had an age $\geq 18$ years. Patients with previous acute coronary syndromes, myocardial revascularization

\footnotetext{
' Department of Cardiology, Santa Marta Hospital, Centro Hospitalar Lisboa Central, EPE, Rua Santa Marta, I 169-024 Lisboa, Portugal

\section{Corresponding Author:}

Ana Teresa Timóteo, Serviço Cardiologia, Hospital Santa Marta, CHLC, EPE, Rua Santa Marta, I 169-024 Lisboa, Portugal

Email: ana_timoteo@yahoo.com
} 
procedure, valvular heart disease, congenital heart disease, or cardiomyopathy were excluded. The local institutional ethics committee approved the study, and all patients gave their written informed consent.

Blood pressure (BP) was measured on several occasions during hospital stay and hypertension was defined by a previous diagnosis of hypertension or the presence of systolic BP $\geq 140 \mathrm{~mm} \mathrm{Hg}$ or diastolic BP $\geq 90 \mathrm{~mm} \mathrm{Hg}$ (mean of 2 consecutive measurements). Patients that smoked during the previous 6 months were classified as smokers and were self-reported. Diabetes was recorded by patient history, raised glucose (fasting level $\geq 126 \mathrm{mg} / \mathrm{dL}$ ), or use of specific therapies.

A venous blood sample was drawn after a 12-hour overnight fast. All the samples were analyzed at the central laboratory of the hospital. Serum glucose, total cholesterol, and triglycerides were determined using automatic standard routine enzymatic methods. High-density lipoprotein (HDL) cholesterol was determined after specific precipitation. Lowdensity lipoprotein (LDL) cholesterol was determined by Friedewald formula.

Coronary angiography was performed by the standard Judkins technique. The coronary angiograms were analyzed using QCA software, Cardiovascular Measurements System (QCA-CMS) version 6.0 (Medis Medical Imaging Systems, Leiden, The Netherlands) by a single operator blinded to the carotid ultrasound results. An automated edge detection algorithm determined vessel centerline and contour and absolute reference vessel and minimum lumen diameters were determined using the calibration factor. Percentage stenosis was calculated from minimum lumen diameter and a normal reference value obtained as an extrapolation of the proximal and distal segments surrounding the stenosis. Significant angiographic CAD was defined as stenosis of $50 \%$ or more in any coronary vessel. The severity of CAD was assessed by the Gensini score, a previously validated method. ${ }^{9}$ The interclass correlation coefficients for intrareader reproducibility were 0.950 for Gensini score and 0.724 to 0.947 for vessel dimension (analyzed for different vessels), which suggests good agreement.

The carotid ultrasound procedure was performed with a Siemens Sonolite system and a 7.5-MHz linear array transducer. The cIMT was measured by a trained radiologist (blinded to the coronary angiographic result) at the distal common carotid artery (CCA; $1 \mathrm{~cm}$ proximal to dilation of the carotid bulb) at the far wall. The higher yield and superior reproducibility of measurement of the CCA IMT compared to internal carotid artery (ICA) and bulb IMT favor its use in the IMT measurements, and this was the segment chosen for the study. Manual measurements were taken and the value of both the right and the left side were obtained for analysis. The final cIMT was obtained as the maximum cIMT between both sides. Plaque was defined as a focal structure encroaching into the arterial lumen with a thickness that is more than $50 \%$ of the surrounding cIMT or more than $1.5 \mathrm{~mm}$. The interclass correlation coefficients for intrareader and interreader reproducibility were 0.907 and 0.820 , respectively, which also suggests good agreement.

\section{Statistical Analysis}

Statistical analysis was conducted using the PASW 18.0 program (SPSS Inc, Illinois, Chicago). Quantitative variables were described as mean and standard deviation or as median values and corresponding 25 th and 75 th percentiles for nonnormally distributed variables and qualitative variables as percentages. Student $t$ test or Mann-Whitney $U$ test was used for between-group comparisons of continuous variables (according to distribution characteristics), while the chi-square test was used for between-group comparisons of categorical variables. Gensini score and some laboratory variables were much skewed, and we performed a 10-based logarithmic transformation $(\log )$ that was used in the subsequent analysis. Pearson correlation was done between cIMT and continuous variables. Bivariate logistic regression models were used to identify predictors of CAD. Multivariate logistic regression analysis (with $\mathrm{CAD}$ as the outcome variable) was performed to determine whether cIMT was an independent predictor of CAD. We used the area under the receiver-operating characteristic (AUC) curve analysis (or C-statistics) to evaluate predictive value for CAD of cIMT and to determine its best cutoff (maximizing the sum of sensitivity and specificity). Several risk prediction models were also considered: (1) established risk factors only, (2) established risk factors plus cIMT, (3) established risk factors plus carotid plaque, and (4) established risk factors plus cIMT plus carotid plaque. We described the AUC for each model to analyze the model predictive capacity.

\section{Results}

We included 300 patients in the study, with a mean age of $64 \pm$ 9 years (aged 38-86 years), 59\% males (Table 1). Significant CAD was present in $51.3 \%$ of patients, but it was significantly lower in females $(35.8 \%$ vs $62.1 \%$; $P<.001)$. Patients with significant $\mathrm{CAD}$ were older, more frequently male and diabetic, had higher levels of blood glucose and triglycerides, and lower levels of HDL cholesterol (Table 1).

In our population, $88 \%$ of the patients had a noninvasive test positive for ischemia. However, even in this group of patients, the prevalence of significant CAD was low, particularly in females (Table 2).

Mean cIMT was $0.88 \pm 0.33 \mathrm{~mm}$, significantly higher in males $(0.94 \pm 0.35$ vs $0.81 \pm 0.29 \mathrm{~mm} ; P=.001)$ and in patients with significant $\mathrm{CAD}(0.96 \pm 0.35$ vs $0.82 \pm 0.29$ $\mathrm{mm} ; P<.001$ ). Carotid plaques were present in $6.0 \%$ of patients.

The cIMT was associated with the number of diseased coronary vessels; however, the only significant difference was found between no diseased vessel and 1 diseased vessel (Figure 1). A more consistent association was found between cIMT and Gensini score, a CAD severity index $(r=.221, P<.001)$.

By receiver-operating curve analysis, we obtained a modest predictive capacity for CAD of isolated cIMT (AUC 0.638, 95\% confidence interval $[\mathrm{CI}] 0.576-0.701 ; P<.001)$, with the best cutoff of $0.85 \mathrm{~mm}$ with a sensitivity of $63 \%$ and specificity of $64 \%$. The same cutoff was obtained in both genders, however 
Table I. Characteristics of the Study Population and by Gender

\begin{tabular}{|c|c|c|c|c|}
\hline Characteristics & $\begin{array}{c}\text { Total } \\
\text { Mean } \pm \text { SD } \\
\text { Median (IQR) } \\
\%\end{array}$ & $\begin{array}{c}\text { CAD } \\
\text { Mean } \pm \text { SD } \\
\text { Median (IQR) } \\
\%\end{array}$ & $\begin{array}{c}\text { No CAD } \\
\text { Mean } \pm \text { SD } \\
\text { Median (IQR) } \\
\%\end{array}$ & $P$ \\
\hline Age (years) & $64.4 \pm 9.2$ & $66.2 \pm 9.2$ & $63.4 \pm 9.9$ & .003 \\
\hline Male gender (\%) & 59 & 7I & 46 & .001 \\
\hline \multicolumn{5}{|l|}{ Risk factors (\%) } \\
\hline Smoking & 9 & II & 8 & .398 \\
\hline Diabetes mellitus & 23 & 29 & 16 & .013 \\
\hline \multicolumn{5}{|l|}{ Laboratorial data } \\
\hline Glucose (mg/dL) & $100(92-115)$ & $104(93-124)$ & $97(91-106)$ & .001 \\
\hline Total cholesterol (mg/dL) & $18 \mid(155-2 \mid 3)$ & $182(158-218)$ & 177 (I52-207) & .208 \\
\hline HDL-cholesterol (mg/dL) & $43(36-54)$ & $4 I(34-50)$ & $47(40-55)$ & $<.001$ \\
\hline
\end{tabular}

Abbreviations: SD, standard deviation; IQR, interquartile range; CAD, coronary artery disease; HDL, high-density lipoprotein; LDL, low-density lipoprotein.

Table 2. Prevalence of Significant Coronary Artery Disease, Analyzing Only Patients With Positive Stress Tests and According to Gender

\begin{tabular}{lcccr}
\hline & Total, \% & Males, \% & Females, \% & $P$ \\
\hline Positive treadmill test & 49.3 & 60.7 & 30.8 & .001 \\
Positive nuclear test & 54.3 & 65.2 & 41.4 & .012 \\
Any positive test & 51.5 & 62.7 & 36.0 & $<.001$ \\
\hline
\end{tabular}

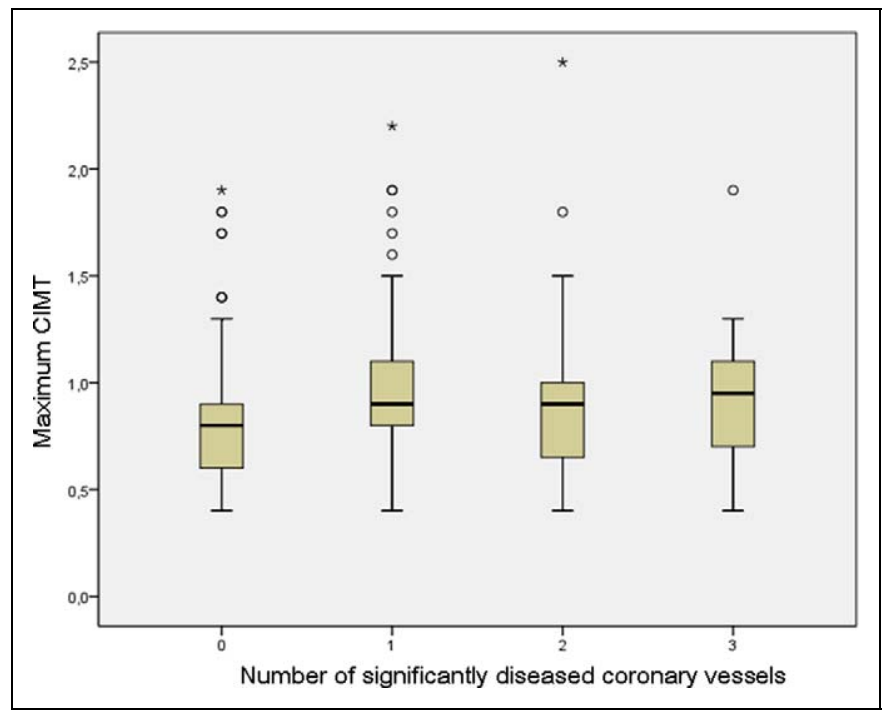

Figure I. Carotid intima-media thickness according to the number of diseased coronary arteries $(P=.003$, post hoc analysis with the only significant difference between no-vessel and I-vessel disease).

with a better AUC for women (Table 3). In a logistic regression model for CAD, cIMT was an independent predictor of CAD, as well as age, gender, and diabetes (Table 4). Even when cIMT was introduced in the model as a categorical value using the previous obtained cutoff, it remained as an independent predictor of CAD (odds ratio 2.26, 95\% CI 1.36-3.75; $P=.002$ ).

To analyze the incremental value of cIMT and carotid plaques over established risk factors, we compared risk prediction models. We found that the AUC for traditional risk factor prediction of CAD was not significantly different from the model that included cIMT, carotid plaque presence, or both (Table 5). However, in women, it was substantially increased by the addition of cIMT or carotid plaque presence, and the combination of risk factors, cIMT, and plaque yielded the highest AUC.

\section{Discussion}

Our study showed a surprisingly low diagnostic yield of coronary angiography. However, recent registries point to the same results. In a 1992 report, between $9 \%$ and $36 \%$ of patients who underwent invasive angiography were found to have normal coronary arteries, but the findings were limited by varying definitions of normal and by different radiographic equipment. ${ }^{10}$ Findings from the Coronary Artery Surgery Study showed that $18.8 \%$ of patients had nonobstructive CAD. ${ }^{11}$ Data from the Society for Cardiac Angiography and Interventions (still more than 15 years old) showed that between $30 \%$ and $35 \%$ of patients had stenosis of less than $50 \% .^{12}$ These data were obtained before the current increase in both noninvasive imaging and the use of cardiac catheterization and include a broad spectrum of patients undergoing angiography, including those with acute coronary syndromes and other emergency indications. Thus, the definition of the patient population is critical in placing our findings in context. The recently published CathPCI Registry of the National Cardiovascular Data Registry (NCDR), a large registry of clinical data and in-hospital outcome data associated with diagnostic cardiac catheterization and percutaneous coronary interventions collected from more than 800 US sites reported also a low diagnostic yield in patients 
Table 3. Evaluation of cIMT Gender-Specific Cutoff Values for the Presence of Significant Coronary Artery Disease by ROC Curve Analysis

\begin{tabular}{lcccccc}
\hline & Cutoff & Se & Sp & AUC & $P$ & RR for CAD (95\% Cl) \\
\hline Females & $0.85 \mathrm{~mm}$ & $64 \%$ & $75 \%$ & $0.715(0.621-0.809)$ & $<.001$ & $5.16(2.33-11.45)$ \\
Males & $0.85 \mathrm{~mm}$ & $63 \%$ & $52 \%$ & $0.557(0.470-0.645)$ & .201 & $1.84(0.99-3.41)$ \\
\hline
\end{tabular}

Abbreviations: ROC, receiver-operating curve; Se, sensitivity; Sp, specificity; AUC, area under curve; RR, relative risk; CAD, coronary artery disease; Cl, confidence interval.

Table 4. Backward Multivariate Logistic Regression Model to Predict Coronary Artery Disease

\begin{tabular}{lrrrr}
\hline & B & Wald & OR $(95 \% \mathrm{Cl})$ & \multicolumn{1}{c}{$P$} \\
\hline Age & 0.033 & 5.442 & I.03 $(1.01-1.06)$ & .020 \\
Male gender & 1.068 & 17.300 & $2.91(1.76-4.81)$ & $<.001$ \\
Diabetes & 0.693 & 5.156 & $2.00(1.10-3.64)$ & .023 \\
cIMT & 0.855 & 4.199 & $2.35(1.04-5.33)$ & .040 \\
\hline
\end{tabular}

Abbreviations: OR, odds ratio; $\mathrm{Cl}$, confidence interval; cMIT, carotid intimamedia thickness.

without previously known CAD, with only a minority of patients with obstructive CAD $(41.0 \%)$ similar to our results. ${ }^{13}$ This registry represents contemporary clinical practice in the community. The NCDR registry also provides insights into patterns of noninvasive testing among patients undergoing diagnostic cardiac catheterization. Previous studies showed that only $41 \%$ of the patients underwent any stress testing before coronary angiography. ${ }^{14}$ The NCDR registry showed that up to $84 \%$ of patients undergoing angiography had undergone a previous noninvasive diagnostic test and a positive result was recorded in $68.6 \%$ of all patients in the cohort. ${ }^{13}$ Similar results were obtained in our population. The increased use of noninvasive testing to rule out ischemia prior to the procedure should result in more effective risk stratification of patients, allowing identification of those patients who would be most likely to benefit from cardiac catheterization and ideally reducing the use of invasive procedures in patients who do not have obstructive disease. Although certain demographic and clinical characteristics could be useful in determining the likelihood that obstructive CAD would be present, the incremental value of a positive result on a noninvasive test was however limited. In NCDR registry, although the association between a positive noninvasive test and the presence of obstructive CAD was significant, the effect of a positive noninvasive test on the ability of the model to predict the presence of obstructive CAD was limited with minimal increase in C-statistic. ${ }^{13}$ It is also important to stress out that patients with chest pain and normal or nonobstructive coronary angiograms are predominantly women, and many have a prognosis that is not as benign as previously thought. ${ }^{15}$ Assessment of endothelial function may help identify patients at risk of future cardiac events. Recent data suggest that ST-segment changes and myocardial reversible perfusion defects in patients with "normal" coronary arteries may be true myocardial ischemia likely related to atherosclerotic disease and not false-positive test results. $^{15}$

Our study confirmed the limited value of noninvasive tests, particularly in women. Thus, there is still need of an inexpensive and easily accessible test that would indicate the likelihood of CAD with reasonable high sensitivity and specificity. A meta-analysis of 8 trials on cIMT, including 37197 asymptomatic individuals who were followed up for a mean of 5.5 years showed that a cIMT increment of $0.1 \mathrm{~mm}$ increased the risk of myocardial infarction by $10 \%$ to $15 \%$ and increased the risk of stroke by $13 \%$ to $18 \%$, after adjusting for age and gender. ${ }^{16}$ Moreover, the cIMT prediction for both events may be better in women than in men and the cIMT prediction may be better for stroke than for CAD when cIMT is measured in the CCA alone. ${ }^{6}$ The fact that cIMT provides additional prognostic information to that of conventional risk factors is pivotal in discussing its clinical utility in primary prevention and was not supported by available data until recently. Since 2000, 7 guidelines or consensus statements recommended measuring cIMT and/or carotid plaque detection as clinical tools to assist cardiovascular disease risk prediction. ${ }^{17}$ However, the US Preventive Services Task Force recently recommended against measurement of anatomical markers of atherosclerosis, including cIMT, basing its negative recommendation regarding the independent predictive value of cIMT for patients at intermediate CAD risk and concerns about this test's ability to reclassify such patients into lower or higher risk categories, therefore altering their clinical management. ${ }^{18,19}$ The very recent report from the Atherosclerosis Risk in Communities (ARIC) study with $>13000$ individuals provide the best evidence, to date, demonstrating the ability of carotid ultrasound data to improve CAD risk prediction. ${ }^{20}$ They found that the AUC for established risk factor prediction of CAD events was significantly increased by the addition of increased cIMT or carotid plaque presence and that the combination of risk factors, cIMT, and plaque yielded the highest AUC. Thus, carotid ultrasoundbased cIMT measurement and identification of plaque presence or absence improves CAD risk prediction and should be considered in the intermediate risk group.

These findings support the previous American Society of Echocardiography's recommendation of combining cIMT and carotid plaque data for optimal risk prediction. ${ }^{17}$ In the ARIC report, although the increments in AUC achieved using the carotid ultrasound data may seem small, they are on the same order of magnitude as the individual contributions of smoking status and systolic BP and greater than the contributions of lipids, family history, and high-sensitivity C-reactive protein. ${ }^{20}$ 
Table 5. Predictive Value for Each Model ${ }^{\mathrm{a}}$

\begin{tabular}{|c|c|c|c|c|}
\hline & Model I & Model 2 & Model 3 & Model 4 \\
\hline \multicolumn{5}{|l|}{ Overall } \\
\hline AUC $(95 \% \mathrm{Cl})$ & $0.70 \mathrm{I}(0.642-0.760)$ & $0.709(0.65 \mathrm{I}-0.768)$ & $0.707(0.649-0.766)$ & $0.709(0.65 \mathrm{I}-0.768)$ \\
\hline$P$ & .613 & .001 & .521 & $<.001$ \\
\hline \multicolumn{5}{|l|}{ Males } \\
\hline AUC $(95 \% \mathrm{Cl})$ & $0.636(0.55 \mathrm{I}-0.720)$ & $0.630(0.547-0.719)$ & $0.638(0.554-0.723)$ & $0.64 \mid(0.557-0.725)$ \\
\hline$P$ & .920 & .519 & .532 & .727 \\
\hline \multicolumn{5}{|l|}{ Females } \\
\hline AUC $(95 \% \mathrm{Cl})$ & $0.689(0.59 \mathrm{I}-0.788)$ & $0.745(0.656-0.834)$ & $0.717(0.62 \mathrm{I}-0.8 \mathrm{I})$ & $0.747(0.658-0.836)$ \\
\hline$P$ & .552 & .887 & .383 & .969 \\
\hline
\end{tabular}

Abbreviations: CIMT, carotid intima-media thickness; $\mathrm{Cl}$, confidence interval; $\mathrm{AUC}$, area under the curve.

a Risk prediction models: (I) established risk factors only; (2) established risk factors plus cIMT; (3) established risk factors plus carotid plaque; (4) established risk factors plus cIMT plus carotid plaque. $P$ values derived using the Hosmer and Lemeshow test.

Indeed, after age and gender inclusion in predictive models, additional risk factors contribute little on an individual basis but are very important when considered together. ${ }^{20}$

In the past few years, several groups have shown that carotid plaque or plaque area are more closely related to $\mathrm{CAD}$ and is more strongly predictive of coronary events. ${ }^{5,8,21-24}$ In fact, established coronary risk factors explain only $15 \%$ to $17 \%$ of cIMT but account for $52 \%$ of the carotid plaque area, suggesting that the carotid plaque area is more representative of atherosclerotic burden than cIMT. ${ }^{25-27}$ Although highly intercorrelated, measurements of cIMT and plaque area probably reflect different biological aspects and stages in the development of atherosclerosis. Whereas plaque is the biological hallmark of atherosclerosis, reflecting subendothelial deposition of cholesterol as well as infiltration of inflammatory cells, a diffuse thickening of the intima-media layer may be caused by other pathogenic mechanisms. In this layer, $20 \%$ is made of the endothelial layer and subendothelial matrix, and the remaining $80 \%$ consists of smooth muscle cells. ${ }^{27}$ Agerelated arterial stiffness and hypertension increase the shear forces acting on the vessel surface, causing hyperplasia of the smooth muscle cells, explaining the association of cIMT with age and hypertension. ${ }^{27}$ Increased cIMT therefore may not necessarily reflect the atherosclerotic process. On the other hand, plaque growth occurs longitudinally along the carotid axis of flow faster than it thickens, which may make plaque area more sensitive as a measure of atherosclerosis. ${ }^{28}$

The present study analyzed directly the relationship between carotid subclinical atherosclerosis and stable significant angiographic coronary artery disease. This end point is different from the most often studied end points since acute cardiovascular events are caused by unstable atherosclerotic plaques, with different pathophysiological mechanisms involved when compared to stable atherosclerotic plaques. Our results showed that cIMT is higher in patients with significant CAD than in patients without CAD. Regression analysis revealed that thickening of the mean intima-media complex more than $0.85 \mathrm{~mm}$ was predictive of significant $\mathrm{CAD}$. As an isolated tool, its predictive capacity for CAD is, however, very modest but slightly better in women. We also found that although in the general population, it was not very helpful in increasing predictive capacity for CAD when added to a predictive model with conventional risk factors, in women there was a substantial incremental value of cIMT to predict significant $\mathrm{CAD}$ and that it was slightly better than carotid plaque. Therefore, cIMT value cannot be used as a sole indicator of CAD but needs to be considered in the panel of studies that is requested, particularly in women who are considered candidates for coronary angiography.

Both cIMT and carotid plaques seemed to have a more profound effect on improving risk prediction in women than in men, and the reason is not completely clear. The most likely explanation is that middle-aged women have a relatively low prevalence of atherosclerosis. For that reason, increased cIMT and plaque presence, which reflects a definite area of atherosclerosis, is more powerful in predicting CAD.

In conclusion, coronary angiography has a low diagnostic yield for significant $\mathrm{CAD}$, particularly in women. In this group, noninvasive tests for risk stratification before coronary angiography also have severe limitations. In women, cIMT seems to be a useful risk stratification tool in association with conventional risk factors for CAD.

\section{Limitations}

Our study is a cross-sectional study, and as such, it is not possible to establish a causal relationship since no information from the follow-up was analyzed and individuals only underwent a single coronary angiogram and carotid ultrasound.

The present scanning protocol for cIMT measurement only focuses on the CCA due to difficulties in adequate evaluation of cIMT in the bulb and ICA. However, scanning of the remaining segments of the carotid arteries for plaques might be important to avoid missing "upstream" advanced atherosclerosis. In fact, we did not account for the potential difference between plaque presence in one artery alone versus in multiple arteries. It is possible that plaque presence in multiple carotid artery segments may be associated with higher risk. Very recent studies showed that cIMT measured solely in the far wall of the CCA is associated with incident stroke in unadjusted analysis, but this association became nonsignificant after adjustment for 
risk factors. ${ }^{29,30}$ However, using measurements that included the carotid bifurcation, where there is a nonlaminar turbulent flow that favors atherosclerotic plaque development, it was associated with stroke but only in women. ${ }^{30}$ This highlights the importance of segment-specific measurements as well as gender influence.

Manual assessment of cIMT is not optimally reproducible. There have been rapid advances in ultrasound technology resulting in greater consistency and resolution of images. Semiautomated edge-detection software to measure cIMT has been developed and has been found to be both accurate and reproducible. This technology might have yielded different results.

This is an angiographic study and as such, coronary plaque morphology was not directly assessed. Intravascular ultrasound allows cross-sectional imaging of coronary arteries and provides more comprehensive assessment of atherosclerotic plaque in vivo and this is considered the gold standard technique. With quantitative coronary angiography analysis, we measured reference diameter and percentage diameter stenosis. However, it has been previously described that this measurement might be misleading because patients with identical measurements in angiography might have different vessel volume and plaque volume when assessed by intravascular ultrasound. In fact, in some patients, vessels might have a compensatory enlargement to prevent building atheroma from encroaching in lumen, thereby concealing the presence of a lesion when angiography is performed. ${ }^{31}$

\section{Declaration of Conflicting Interests}

The authors declared no potential conflicts of interest with respect to the research, authorship, and/or publication of this article.

\section{Funding}

The authors received no financial support for the research, authorship, and/or publication of this article.

\section{References}

1. The European Health Report 2009. Health and Health Systems. Copenhagen, Denmark: World Health Organization Regional Office for Europe; 2009.

2. World Health Organization on behalf of the European Observatory of Health Systems and Policies. Health in the European Union. Trends and Analysis. Copenhagen, Denmark: World Health Organization Regional Office for Europe; 2009.

3. Simon A, Gariepy J, Chironi G, Megnien JL, Levenson J. Intimamedia thickness: a new tool for diagnosis and treatment of cardiovascular risk. J Hypertens. 2002;20(2):159-169.

4. Chambless LE, Heiss G, Folsom AR, et al. Association of coronary heart disease incidence with carotid arterial wall thickness and major risk factors: the Atherosclerosis Risk in Communities (ARIC) study 1987-1993. Am J Epidemiol. 1997;146(6):483-494.

5. O'Leary DH, Polak JF, Kronmal RA, Manolio TA, Burke GL, Wolfson SK Jr. Carotid-artery intima and media thickness as a risk factor for myocardial infarction and stroke in older adults: Cardiovascular Health Study Collaborative Research Group. $N$ Engl J Med. 1999;340(1):14-22.
6. Simon A, Megnien JL, Chironi G. The value of carotid intimamedia thickness for predicting cardiovascular risk. Arterioscler Thromb Vasc Biol. 2010;30(2):182-185.

7. Rosvall M, Janzon L, Berglund G, Engstrom G, Heldblad B. Incidence of stroke is related to carotid IMT even in the absence of plaque. Atherosclerosis. 2005;179(2):325-331.

8. Ebrahim S, Papacosta O, Whincup P, et al. Carotid plaques, intima media thickness, cardiovascular risk factors, and prevalent cardiovascular disease in men and women: the British regional heart study. Stroke. 1999;30(4):841-850.

9. Gensini GG. A more meaningful scoring system for determining the severity of coronary heart disease. Am J Cardiol. 1983;51(3): 606.

10. Bernstein SJ, Laouri M, Hilborne LH, et al. Coronary Angiography: A Literature Review and Rating of Appropriateness and Necessity. Santa Monica, CA: RAND; 1992.

11. Kemp HG, Kronmal RA, Vlietsra RE, Frye RL. Seven-year survival of patients with normal or near-normal coronary arteriograms: a CASS registry study. J Am Coll Cardiol. 1986;7(3): 479-483.

12. Johnson LW, Krone R. Cardiac cathetherization 1991: a report of the Registry of the Society for Cardiac Angiography and Interventions (SCA\&I). Cather Cardiovasc Diagn. 1993;28(3):219-220.

13. Patel MR, Peterson ED, Dai D, et al. Low diagnostic yield of elective coronary angiography. $N$ Engl J Med. 2010;362(10):886-895.

14. Lin GA, Dudley RA, Lucas FL, Malenka DJ, Vittinghoff E, Redberg RF. Frequency of stress testing to document ischemia prior to elective percutaneous coronary intervention. JAMA. 2008;300(15):1765-1773.

15. Bugiardini R, Merz CB. Angina with "normal" coronary arteries. A changing philosophy. JAMA. 2005;293(4):477-484.

16. Lorenz MW, Markus HS, Bots ML, Rosvall M, Stizer M. Prediction of clinical cardiovascular events with carotid intima-media thickness: a systematic review and meta-analysis. Circulation. 2007;115(4):459-467.

17. Stein JH, Korcarz CE, Hurst T, et al. Use of carotid ultrasound to identify subclinical vascular disease and evaluate cardiovascular disease risk: a consensus statement from the American Society of Echocardiography Carotid Intima-media Thickness Task Force. Endorsed by the Society for Vascular Medicine. J Am Soc Echocardiography. 2008;21(2):93-111.

18. United States Preventive Services Task Force. Using nontraditional risk factors in coronary heart disease risk assessment. U. S. Preventive Services Task Force Reccomendation Statement. Ann Intern Med. 2009;151:474-482.

19. Helfand M, Bucley DI, Freeman M, et al. Emerging risk factors for coronary heart disease: a summary of systematic reviews conducted for the U.S. Preventive Services Task Force. Ann Intern Med. 2009;151(7):496-507.

20. Nambi V, Chambless L, Folsom AR, et al. Carotid intima-media thickness and presence or absence of plaque improves prediction of coronary heart disease risk: the ARIC (Atherosclerosis Risk In Communities) study. J Am Coll Cardiol. 2010;55(15):1600-1607.

21. Rosvall M, Janzon L, Berglund G, Engstrom G, Heldblad B. Incident coronary events and case fatality in relation to common carotid intima-media thickness. J Intern Med. 2005;257(5):430-437. 
22. Chan SY, Mancini GB, Kuramoto L, Schulzer M, Frohlich J, Ignaszewski A. The prognostic importance of endothelial dysfunction and carotid atheroma burden in patients with coronary artery disease. J Am Coll Cardiol. 2003;42(6):1037-1043.

23. Johnsen SH, Mathiesen BB, Joakimsen O, et al. Carotid atherosclerosis is a stronger predictor of myocardial infarction in women than in men: a 6-year follow-up study of 6226 persons: the Tromso study. Stroke. 2007;38(11):2873-2880.

24. Spence ID, Eliasziw M, Di Cicco M, Hackam DG, Galil R, Lohmann T. Carotid plaque area: a tool for targeting and evaluating vascular preventive therapy. Stroke. 2002;33(12):2916-2922.

25. O'Leary DH, Polak JF, Kronmad RA, et al. Cardiovascular Health Study Collaborative Research Group. Thickening of the carotid wall: a marker for atherosclerosis in the elderly? Stroke. 1996; 27(2):224-231.

26. Al-Shali K, House AA, Hanley AJ, et al. Differences between carotid wall morphological phenotypes measured by ultrasound in one, two and three dimensions. Atherosclerosis. 2005;178(2): 319-325.
27. Finn AV, Kolodgie FD, Virmani R. Correlation between carotid intimal/medial thickness and atherosclerosis: a point of view from pathology. Arterioscler Thromb Vasc Biol. 2010;30(2): 177-181.

28. Barnett PA, Spence JD, Manuck SB, Jennings JR. Physiological stress and the progression of carotid artery disease. J Hypertens. 1997; 15(1):49-55.

29. Mathiesen EB, Johnsen SH, Wilsgaard T, Bonaa KH, Loochen ML, Njolstad I. Carotid plaque area and intima-media thickness in prediction of first-ever ischemic stroke: a 10-year follow-up of 6584 men and women: the Tromson study. Stroke. 2011; 42(4):972-978.

30. Polak JF, Pencina MJ, O'Leary DH, D'Agostino RB. Common carotid artery intima-media thickness progression as a predictor of stroke in Multi-Ethnic Study of Atherosclerosis. Stroke. 2011;42:3017-3021.

31. Amano T, Matsubara T, Uetani T, et al. Impact of metabolic syndrome on tissue characteristics of angiographically mild to moderate coronary lesions. J Am Coll Cardiol. 2007;49(11):1149-1156. 
Copyright of Angiology is the property of Sage Publications Inc. and its content may not be copied or emailed to multiple sites or posted to a listserv without the copyright holder's express written permission. However, users may print, download, or email articles for individual use. 